\title{
THE FORMATION OF THE DEAD CORE IN PARABOLIC REACTION-DIFFUSION PROBLEMS
}

\author{
BY
}

\author{
CATHERINE BANDLE AND IVAR STAKGOLD
}

\begin{abstract}
For some nonlinear parabolic problems of reaction-diffusion, a region of zero reactant concentration may be formed in finite time. Conditions are formulated for the existence of such a dead core and estimates for its time of onset are also given. These results complement previous ones that dealt with the stationary (elliptic) problem.
\end{abstract}

\section{Introduction.}

1.1. In a simple model for a reaction-diffusion process, the nondimensional concentration $c$ and nondimensional temperature $v$ satisfy the equations $[\mathbf{A r}]$

$$
\begin{cases}c_{t}-\Delta c+\mu^{2} F(c) \exp \left[\gamma\left(\frac{v-1}{v}\right)\right]=0 & \text { in } D \times \mathbf{R}^{+}, \\ \frac{1}{L} v_{t}-\Delta v-\nu \mu^{2} F(c) \exp \left[\gamma\left(\frac{v-1}{v}\right)\right]=0 & \text { in } D \times \mathbf{R}^{+},\end{cases}
$$

and are subject to the boundary and initial conditions

$$
\left\{\begin{array}{l}
c(x, t)=v(x, t)=1 \quad \text { on } \partial D \times \mathbf{R}^{+} \\
c(x, 0)=c_{0}(x), \quad v(x, 0)=v_{0}(x)
\end{array}\right.
$$

Here $D \subset \mathbf{R}^{N}$ is the domain where the reaction takes place, $\mu, \nu, \gamma, L$ are dimensionless parameters $(\mu, \gamma, L$ are positive).

The first equation in $(1.1)^{\prime}$ describes the consumption of the reactant whose concentration $c$ would decay to zero if it were not for the boundary condition, whose effect is to replenish the reactant in $D$. The second equation is obtained from a heat balance. Here $\nu$ is a heat of reaction which is positive if the reaction is exothermic, negative if it is endothermic.

If $\nu=0$ and $v_{0}(x)=1$, then $v(x, t) \equiv 1$ and the system is reduced to a scalar equation for $c$. Such a reduction is also possible if $L=1$ (equal diffusivities for mass and heat transfer), for then the function $w=\nu c+v$ satisfies

$$
\begin{gathered}
w_{t}-\Delta w=0 \quad \text { in } D \times \mathbf{R}^{+}, \quad w=\nu+1 \text { on } \partial D \times \mathbf{R}^{+}, \\
w(x, 0)=\nu c_{0}(x)+v_{0}(x)
\end{gathered}
$$

and is uniquely determined. As $t \rightarrow \infty, w$ tends to the value $\nu+1$. Taking the initial condition on $w$ equal to this asymptotic limit, we see that $w \equiv \nu+1$ for all $x, t$. Therefore, if $L=1$ and $v_{0}(x)=\nu+1-\nu c_{0}(x), c(x, t)$ satisfies the scalar problem

(1.2) $c_{t}-\Delta c+\lambda G(c)=0 \quad$ in $D \times \mathbf{R}^{+}, \quad c=1$ on $\partial D \times \mathbf{R}^{+}, \quad c(x, 0)=c_{0}(x)$,

Received by the editors September 8, 1983.

1980 Mathematics Subject Classification. Primary 35K60; Secondary 35J65, 35B40, 35R99. 
where $0 \leq c_{0}(x) \leq 1, \lambda>0$, and

$$
G(c)=F(c) \exp \left[\gamma \frac{\nu(1-c)}{1+\nu(1-c)}\right] .
$$

It is clear that the isothermal problem can be regarded as a special case of (1.2) with $\nu=0$ (and hence $G=F$ ).

Usually $F(c)$, which is originally defined only for $c \geq 0$, is of the form $c^{p}$ where $p \geq 0$ is the order of the reaction. Of course, $F$ must vanish when $c=0$ since no reaction can take place when the concentration of the reactant is zero. If $p=0$, then $F$ will be discontinuous at $c=0$; if $0<p<1, F$ is continuous at $c=0$ but has an infinite derivative there. If the reaction is endothermic $(\nu<0), G(c)$ has properties similar to those of $F(c)$. We shall confine ourselves in this paper to endothermic or isothermal reactions $(\nu \leq 0)$.

From the maximum principle, it then follows that $c \leq 1$. We are looking for solutions of (1.2) which satisfy $c \geq 0$; by extending the definition of $F$ to be zero for $c<0$, we insure that every solution of (1.2) does, in fact, satisfy $c \geq 0$.

If $0 \leq p<1$, the solution of the stationary problem corresponding to (1.2) will, for sufficiently large $\lambda$, vanish identically in a closed region known as a dead core or dead zone (see [Ba-Sp-St, Fr-Ph, Di-He, 1, St]). If $p \geq 1$, there is never a dead core. The existence of a dead core may at first seem surprising since the reactant concentration is maintained at a constant positive value on the boundary. The physical explanation is that if the reaction rate remains high as the concentration decreases, diffusion may not be strong enough to draw the reactant from the boundary into the central part of $D$. Does a dead core also exist for the time-dependent problem? If so, how does it grow in time? We shall find that whenever the stationary problem has a dead core, so does the time-dependent problem. We shall study qualitatively and quantitatively the onset and development of the dead core as a function of time. A very general version of the time-dependent problem with emphasis on the zeroth-order reaction is presented in an unpublished paper [Di-He, 2], where maximal monotone operator techniques are used. Our aim is to extend the results in $[\mathbf{B a}-\mathbf{S p}-\mathbf{S t}]$ to the parabolic problem. The main tools will be maximum principles, the method of upper and lower solutions, and rearrangement techniques. We do not hesitate at times to sacrifice some generality to obtain more concrete results. For instance, we shall only consider the case of initial concentration $c_{0}(x)=1$, which corresponds to the slowest formation of the dead core.

For convenience, we set $u=1-c$ and study the problem for $u$. Since no additional difficulties arise, we shall replace the Laplacian by an elliptic operator in divergence form.

We now give the precise formulation of the problem considered in this paper.

1.2. Let

$$
A=\sum_{i, j=1}^{N} \frac{\partial}{\partial x_{i}}\left(a_{i j}(x) \frac{\partial}{\partial x_{j}}\right)
$$

be a uniformly elliptic operator such that $a_{i j}=a_{j i} \in C^{1}(\bar{D})$ and

$$
\sum_{i, j=1}^{N} a_{i j}(x) \xi_{i} \xi_{j} \geq \sum_{i=1}^{N} \xi_{i}^{2}, \quad \forall \xi \in \mathbf{R}^{N} \text { and } \forall x \in \bar{D} .
$$


Problem (P) is defined as follows:

$$
u_{t}-A u=\lambda g(u) \quad \text { in } D \times \mathbf{R}^{+}, \lambda \in \mathbf{R}^{+},
$$

with the boundary and initial conditions

$$
u=0 \quad \text { in } \partial D \times \mathbf{R}^{+}, \quad u(x, 0) \equiv 0 .
$$

Based on the discussion following equation (1.2), the function $g: \mathbf{R} \rightarrow \mathbf{R}^{+}$is subject to the following conditions:

(A-1) $g(\sigma)$ is positive, and continuously differentiable on $(-\infty, 1)$ and continuous on $R$.

(A-2) $g(\sigma)$ is nonincreasing in $(-\infty, 1)$.

$(\mathrm{A}-3) g(0)=1$.

(A-4) $g(\sigma)=0$ for $\sigma \geq 1$.

$(\mathrm{A}-5) g(\sigma)=g_{0}(\sigma)(1-\sigma)_{+}^{p}$ for some $p, 0<p<1$, with $g_{0}(1-)>0$ and $g_{0}^{\prime}(1-)$ finite. Here the notation $a_{+}$indicates the larger of $a$ and 0 .

Condition (A-5) could be relaxed somewhat, but is all that is needed for most problems physical interest. The requirement $0<p<1$ anticipates that no dead core can exist for the time-dependent problem if $p \geq 1$. Condition (A-2) holds whenver the process is endothermic or isothermal. Some of our results carry over to the exothermic case (when $g$ is not necessarily monotonically increasing) and to the case $p=0$ (when $g$ is discontinuous at $\sigma=1$ ), but we shall not always attempt to specify when such generalizations are possible.

It is clear that any solution of problem (P) satisfies $0 \leq u \leq 1$. Since $g$ is not necessarily differentiable at $\sigma=1$, problem (P) might not have a classical solution. The concept of a weak solution, which will be used here, stems from a variational approach to problem (P). More details are found in [De-He, Tr].

Let us write $V$ for the space $L^{q}\left(0, T ; W^{1, q}(D)\right), q \geq 2, V^{\prime}$ for its dual and $V_{0}$ for $L^{q}\left(0, T ; W_{0}^{1, q}(D)\right)$.

According to [De-He], $u$ is a weak solution of $(\mathrm{P})$ in $(0, T)$ if $u \in V_{0}, u_{t} \in V^{\prime}$, $u(\cdot, t) \rightarrow 0$ in $L^{2}(D)$ as $t \rightarrow 0+$ and

$$
\begin{gathered}
\int_{0}^{T} d \tau \int_{D} u_{t} v d x+\int_{0}^{T} d \tau \int_{D_{i, j=1}} \sum_{i j}^{N} a_{x_{i}} v_{x_{j}} d x \\
=\lambda \int_{0}^{T} d \tau \int_{D} g(u) v d x \quad \forall v \in V_{0} .
\end{gathered}
$$

We shall refer to $u$ as a classical solution if $u, u_{t}$ and $u_{x_{i} x_{j}}$ are continuous and if it solves $(\mathrm{P})$ in the classical sense.

LemMa 1.1. Problem (P) has at most one solution.

ProOF. Suppose there are two different solutions $u_{1}$ and $u_{2}$. We may then assume $u_{1}<u_{2}$ in $Q_{0} \subseteq D \times(0, T)$. The function

$$
\delta:= \begin{cases}u_{2}-u_{1} & \text { in } Q_{0}, \\ 0 & \text { elsewhere }\end{cases}
$$


belongs to $V_{0}$ and is nonnegative. From (1.4), together with the monotonicity condition (A-2), we conclude that

$$
\frac{1}{2} \int_{0}^{T} \frac{d}{d t}\left[\int_{D} \delta^{2}(x, t) d x\right] d t+\int_{Q_{0}}\left(\sum_{i, j=1}^{N} a_{i j} \delta_{x_{i}} \delta_{x_{j}}\right) d x d t \leq 0
$$

Observe that

$$
\phi(t):=\int_{D} \delta^{2}(x, t) d x
$$

belongs to $W^{1,2}(0, T)$ and is therefore absolutely continuous, and thus

$$
\int_{0}^{T} \dot{\phi} d t=\phi(T)
$$

This combined with (1.5) implies that $\delta=0$ in $Q_{0}$, contradicting our assumption.

DEFINITION. The set

$$
\Omega(t)=\{x \in D: u(x, t)=1\}
$$

will be called the dead core of problem (P) at time $t$. Recall that $u=1$ corresponds to the concentration $c=0$ in the original problem.

1.3. Our paper is organized as follows. In $\S 2$ we collect results on the existence and regularity of the solution and study its behavior as a function of time. The methods employed in this part are standard. In $\S 3$ we deal with the dead core $\Omega(t)$. We derive monotonicity properties for $\Omega(t)$, estimates for the time of onset, size and location of the dead core. The principal results are contained in Corollary 3.2 and Theorems 3.2-3.4. In $\S 4$ we use rearrangement methods (level-surface analysis) to derive isoperimetric inequalities for $\max _{x \in D} u(x, t)$ and meas $\Omega(t)$ which depend only on meas $D$ and which are expressed in terms of the solution of the symmetric problem. This problem corresponds to (P) with $A$ replaced by $\Delta$ and $D$ by a ball of equal measure.

2. The method of upper and lower solutions and comparison results.

2.1. The main tool of this section is an existence theorem of Deuel and Hess [De-He] based on the concept of upper and lower solutions.

Definition. A function $\underline{u} \in V$ with $\underline{u}_{t} \in V^{\prime}$ is called a lower solution of (P) if

$$
\underline{u}(x, t) \leq 0 \quad \text { in } \partial D \times(0, T), \quad \underline{u}(x, 0) \leq 0 \quad \text { in } D
$$

and

$$
\begin{aligned}
\int_{0}^{T} d \tau \int_{D} \underline{u}_{t} v d x+\int_{0}^{T} d \tau \int_{D} \sum_{i, j=1}^{N} a_{i j} \underline{u}_{x_{i}} v_{x_{j}} d x \leq \lambda \int_{0}^{T} d \tau \int_{D} g(\underline{u}) v d x & \text { for all nonnegative } v \in V_{0}
\end{aligned}
$$

(in short, $\underline{u}_{t}-A \underline{u} \leq \lambda g(\underline{u})$ in $D \times \mathbf{R}^{+}$). Conversely, $\bar{u}$ is an upper solution, if the inequality signs are reversed.

The method developed in $[\mathrm{De}-\mathrm{He}]$ to treat problems with periodic solutions and variational inequalities can easily be adjusted to establish an existence and comparison theorem for problem (P). 
LEMMA 2.1. If there exist upper and lower solutions in $(0, T)$ such that $\underline{u} \leq \bar{u}$ in $D \times(0, T)$, then $(\mathrm{P})$ has a weak solution with

$$
\underline{u}(x, t) \leq u(x, t) \leq \bar{u}(x, t) \quad \text { in } D \times(0, T) .
$$

The existence of a weak positive solution of (P) for all $t>0$ now follows immediately by taking $\underline{u} \equiv 0$ as a lower, and $\bar{u} \equiv 1$ as an upper, solution.

For our investigations we shall often use a weaker form of Lemma 2.1, namely

LEMMA 2.1'. If $\bar{u}$ is a nonnegative upper solution of $(\mathrm{P})$, then $u(x, t) \leq \bar{u}(x, t)$ a.e. Similarly, if $\underline{u} \leq 1$ is a lower solution, then $\underline{u}(x, t) \leq u(x, t)$ a.e.

The classical maximum principle for parabolic equations $[\mathbf{P r}-\mathbf{W e}]$ will play a major role. We shall need it in the following form.

LEMMA 2.2. Let $v, v_{t}, v_{x_{i} x_{j}}$ be continuous and satisfy $v_{t}-A v \geq 0$ in some bounded domain $B \subseteq D \times \mathbf{R}^{+}$. If $v$ assumes its minimum $m$ at an interior point $P_{0} \in B$, then $v=m$ on any continuous curve $x=x(\sigma), t=t(\sigma)\left(0 \leq \sigma \leq \sigma_{0}\right)$, contained in $B$ such that $t(\sigma)$ increases as the curve is traced from $P$ to $P_{0}$.

After these preliminaries we are in position to derive some qualitative properties of the solution $u$ of problem (P).

LEMMA 2.3. (i) $u(x, \cdot)$ increases monotonically to the solution $u(x)$ of the stationary problem

(ii) Let $x \in D-\Omega\left(t_{0}\right)$ for $t_{0}>0$; then $u$ is a classical solution in $\left\{D-\Omega\left(t_{0}\right)\right\} \times$ $\left[0, t_{0}\right]$.

(iii) If $x \in D-\Omega\left(t_{0}\right)$, then $u\left(x, t_{2}\right)<u\left(x, t_{1}\right)$ for $t_{2}<t_{1} \leq t_{0}$.

PROOF. (i) Consider the function $v(x, t):=u(x, t+\Delta t)$ for some $\Delta t>0$. It is a positive solution of $\left(\mathrm{P}^{\prime}\right)$, vanishes in $\partial D \times \mathbf{R}^{+}$and satisfies $v(x, 0)=u(x, \Delta t) \geq 0$. Thus, $v$ is an upper solution and, by Lemma $2.1^{\prime}$, we have

$$
u(x, t) \leq v(x, t)=u(x, t+\Delta t) .
$$

For the second part of (i), we refer to the standard literature.

(ii) Because of (i), the fact that $x \in D-\Omega\left(t_{0}\right)$ implies that $x \in D-\Omega(t)$ for all $t<t_{0}$. From Lemma 2.1 and the classical regularity theorems for elliptic equations, we conclude that $\forall q \geq 2, u \in W^{2,2}(K) \cap W^{1, q}(K)$ for every compact set $K \in D-\Omega\left(t_{0}\right)$. Moreover, we have $u(\cdot, t)<1$ in $K$, so that by $(\mathrm{A}-1)$ the equation $u_{t t}-A u_{t}=\lambda g^{\prime}(u) u_{t}$ in $K \times\left(0, t_{0}\right)$ is meaningful. Subsequent application of Theorem 42.1 of [ $\mathbf{T r}$ ] and a bootstrap argument yield the desired result.

Assertion (iii) will be proved by contradiction. Suppose there exists a point $x_{0} \in D-\Omega\left(t_{0}\right)$ such that $u\left(x_{0}, t_{0}\right)=u\left(x_{0}, t_{0}-\Delta t\right)$ for some $\Delta t>0$. Let $B\left(x_{0}, \rho\right):=$ $\left\{x:\left\|x-x_{0}\right\|<\rho\right\}$ be a ball strictly contained in $D-\Omega\left(t_{0}\right)$, and set

$$
C:=B\left(x_{0}, \rho\right) \times\left(\Delta t, t_{0}\right) \text { and } Z:=B\left(x_{0}, \rho\right) \times\left(0, t_{0}\right) .
$$

By (ii), $u(x, t)$ and $u(x, t-\Delta t)$ are classical solutions of problems $(\mathrm{P})^{\prime}$ in $C$ and their difference

$$
d(x, t):=u(x, t)-u(x, t-\Delta t)
$$

is nonnegative in $\bar{C}$ where it satisifes

$$
d_{t}-A d=\lambda[g(u(x, t))-g(u(x, t-\Delta t))] \geq c_{0} d, \quad c_{0}=\inf _{Z}\left\{\lambda g^{\prime}(u(x, t))\right\} .
$$


Note that $\left|c_{0}\right|<\infty$. The maximum principle applied to $e^{-c_{0} t} d$ yields $d \equiv 0$ in $C$ and thus $u_{t} \equiv 0$ in $C$. By (ii), $u$ is a classical solution of $(\mathrm{P})^{\prime}$ in $Z$ and, consequently, $v=u_{t}$ is differentiable and satisfies

$$
v_{t}-A v=\lambda g^{\prime}(u) v \geq c_{0} v \quad \text { in } Z, \quad v \geq 0 \quad \text { in } Z, \quad v \equiv 0 \quad \text { in } C .
$$

The same argument as above implies $v \equiv 0$ in $Z$, which is impossible.

LEMMA 2.4. (i) If $u_{k}(k=1,2)$ is the solution of (P) with $\lambda g=\lambda_{k} g_{k}$ and $\lambda_{1} g_{1}(\sigma) \leq \lambda_{2} g_{2}(\sigma) \forall \sigma \in \mathbf{R}^{+}$, then $u_{2}(x, t) \geq u_{1}(x, t)$ in $D \times \mathbf{R}^{+}$.

(ii) If $u_{1}$ and $u_{2}$ are the solutions of (P) in $D_{1}$ and $D_{2}$, and if $D_{1} \subseteq D_{2}$, then $u_{2}(x, t) \geq u_{1}(x, t)$ in $D_{1} \times \mathbf{R}^{+}$.

PROOF. $u_{2}$ is an upper solution for the problem for $u_{1}$.

Lemma 2.4 enables us to compare the solution for an arbitrary domain $D$ with those for an inscribed ball, for a circumscribed ball or for a slab containing $D$. Such comparisons are quite useful for the stationary problem because explicit solutions are sometimes available for the ball and slab. Unfortunately, this is not the case for the parabolic problem.

There are some simple upper and lower solutions to the parabolic problem that immediately come to mind. Obviously, $u \equiv 0$ is a lower solution and $u \equiv 1$ is an upper solution. The solution $u(x)$ of the stationary problem is also an upper solution. The solution of the ordinary differential equation

$$
d z / d t=\lambda g(z(t)), \quad z(0)=0
$$

is obviously an upper solution. It should be noted that $z$ is the exact solution of the partial differential equation $(P)^{\prime}$ with the Neumann boundary condition of vanishing normal derivative. Under this boundary condition, no reactant can enter $D$, so the concentration $1-z$ must drop off faster than $1-u$ and, hence, $z(t)$ must exceed $u(x, t)$. Specializing to a $p$ th-order isothermal reaction, $g(\sigma)=(1-\sigma)_{+}^{p}$, we have

$$
z(t)= \begin{cases}1-\{1-\lambda(1-p) t\}^{1 /(1-p)}, & t<[\lambda(1-p)]^{-1} \\ 1, & t \geq[\lambda(1-p)]^{-1}\end{cases}
$$

2.2. Beginning with either an upper or lower solution, it is possible to set up an alternating iteration scheme which will converge to the solution of $(P)$. We first need

LEMMA 2.5. Let $\bar{u}$ be any upper solution of $(\mathrm{P})$ and let $\psi$ solve

$$
\psi_{t}-A \psi=\lambda g(\bar{u}) \quad \text { in } D \times \mathbf{R}^{+}, \quad \psi=0 \quad \text { in } \partial D \times \mathbf{R}^{+}, \quad \psi(x, 0)=0 \text {. }
$$

Then $\psi$ is a lower solution of $(\mathbf{P})$. Conversely, if $\bar{u}$ is replaced by a lower solution, then the solution of (2.2) yields an upper solution.

PROOF. According to the definition of an upper solution,

$$
\begin{gathered}
\psi_{t}-A \psi=\lambda g(\bar{u}) \leq \bar{u}_{t}-A \bar{u} \quad \text { in } D \times \mathbf{R}^{+} \\
\psi=0 \leq \bar{u} \quad \text { in } \partial D \times \mathbf{R}^{+}, \quad \psi(x, 0)=0 \leq \bar{u}(x, 0) .
\end{gathered}
$$

Suppose $\psi-\bar{u} \geq 0$ in $B \subseteq D \times \mathbf{R}^{+}$; then

$$
v= \begin{cases}\psi-\bar{u} & \text { in } B \\ 0 & \text { elsewhere }\end{cases}
$$


belongs to $V_{0}$ and is nonnegative. Hence

$$
\frac{1}{2} \int_{D \cap B}(\psi(x, T)-\bar{u}(x, T))^{2} d x+\int_{B \cap\{t<T\}} \sum_{i, j=1}^{N} a_{i j}(\psi-\bar{u})_{x_{i}}(\psi-\bar{u})_{x_{j}} d x \leq 0
$$

which proves $\psi \leq \bar{u}$ and, hence, by (A-2), $\psi_{t}-A \psi=\lambda g(\bar{u}) \leq \lambda g(\psi)$. The second part of the lemma is established in the same way.

Consider now the sequence $\left\{u_{n}\right\}_{n=1}^{\infty}$ defined by means of the Picard iteration

$$
\begin{gathered}
\partial u_{n} / \partial t-A u_{n}=\lambda g\left(u_{n-1}\right) \quad \text { in } D \times \mathbf{R}^{+}, \\
u_{n}=0 \quad \text { in } \partial D \times \mathbf{R}^{+}, \quad u_{n}(x, 0)=0, \quad n=1,2 .
\end{gathered}
$$

LEMMA 2.6. (i) If we start with a lower solution $u_{0}=\underline{u}$ and if $u_{2} \geq u_{0}$, then $\underline{u}=u_{0} \leq u_{2} \leq u_{4} \leq \cdots \leq u_{5} \leq u_{3} \leq u_{1}$.

(ii) Conversely, if $u_{0}=\bar{u}$ and $u_{2} \leq u_{0}$, then $u_{1} \leq u_{3} \leq u_{5} \leq \cdots \leq u_{4} \leq u_{2} \leq$ $u_{0}=\bar{u}$.

ProOF. By Lemma 2.5 we have $u_{1} \geq u_{0}=\underline{u}$. Moreover,

$$
\left(u_{1}\right)_{t}-A u_{1}=\lambda g\left(u_{0}\right) \geq \lambda g\left(u_{1}\right)=\left(u_{2}\right)_{t}-A u_{2} .
$$

Hence

$$
u_{1} \geq u_{2} \geq u_{0} \quad \text { and } \quad \lambda g\left(u_{1}\right) \leq\left(u_{3}\right)_{t}-A u_{3}=\lambda g\left(u_{2}\right) \leq \lambda g\left(u_{0}\right)
$$

which implies $u_{2} \leq u_{3} \leq u_{1}$. By induction we get (i). Similarly, we prove (ii).

For instance, the lemma applies if $\underline{u}=0$ and $\bar{u}=u(x)$, the solution of the stationary problem.

Although Lemma 2.6 gives us a recipe to construct a sequence converging to $u(x, t)$, it is not useful in determining whether or not a dead core is present in finite time. This sequence of linear problems often leads to solutions that only get exponentially close to the asymptotic limit without actually coinciding in finite time with any part of the expected dead core.

2.3. In $\S 4$ it will be convenient to consider, instead of problem (P), a perturbation of the type

$$
u_{t}^{\varepsilon}-A u^{\varepsilon}=\lambda g_{\varepsilon}\left(u^{\varepsilon}\right) \quad \text { in } D \times \mathbf{R}^{+}
$$

subject to the boundary and initial conditions $(\mathrm{P})^{\prime \prime}, g_{\varepsilon}(\sigma)$ being continuously differentiable in $(0,1)$, strictly decreasing, $g_{\varepsilon}(\sigma) \geq g(\sigma)$ in $(0,1)$ and $g_{\varepsilon}(\sigma)-g(\sigma)<\varepsilon$ in $(0,1)$.

From Lemma 2.4(i) it follows that $u^{\varepsilon} \geq u$. Moreover, we have

LEMMA 2.7. Under the conditions mentioned above,

$$
\lim _{\varepsilon \rightarrow 0} u^{\varepsilon}(x, t)=u(x, t)
$$

uniformly in every compact subset of $D \times \mathbf{R}^{+}$.

ProOF. Let $G(x, t ; y, \tau)$ be the Green's function corresponding to the operator $\partial / \partial t-A$ and vanishing on the boundary. Since $u^{\varepsilon}$ and $u$ are continuous, their difference satisfies

$$
u^{\varepsilon}(x, t)-u(x, t)=\lambda \int_{0}^{t} \int_{D} G(x, t ; y, \tau)\left[g_{\varepsilon}\left(u^{\varepsilon}\right)-g(u)\right] d y d \tau \geq 0
$$


Furthermore,

$$
g_{\varepsilon}\left(u^{\varepsilon}\right)-g(u)=\left[g_{\varepsilon}\left(u^{\varepsilon}\right)-g_{\varepsilon}(u)\right]+\left[g_{\varepsilon}(u)-g(u)\right] \leq \varepsilon,
$$

and thus,

$$
u^{\varepsilon}(x, t)-u(x, t) \leq \lambda \varepsilon C(T) \quad \text { for } t \leq T, x \in \bar{D} .
$$

\section{The formation of the dead core.}

3.1. In this part we derive comparison theorems for the dead core. From Lemma 2.4 we immediately get

COROLlaRY 3.1. (i) If $\tilde{D} \supset D$, then $\tilde{\Omega}(t) \supseteq \Omega(t)$.

(ii) If $\tilde{\lambda} \tilde{g} \geq \lambda g$, then $\tilde{\Omega}(t) \supseteq \Omega(t)$.

Since $u(x, \cdot)$ is nondecreasing, we have

THEOREM 3.1. For any $\Delta t>0$, we have $\Omega(t-\Delta t) \subseteq \Omega(t)$. In addition, $\operatorname{dist}(\partial \Omega(t), \Omega(t-\Delta t))>0$.

PROOF. The first statement is a consequence of Lemma 2.3(i). The proof of the second statement is patterned after [Fr-Ph, Theorem 1.6]. ${ }^{1}$ Suppose the assertion is false. Then there exists a point $x_{0} \in \partial \Omega\left(t_{0}-\Delta t\right) \cap \partial \Omega\left(t_{0}\right) . \Omega\left(t_{0}\right)$ may consist of several components. We shall focus on the one, say $\tilde{\Omega}\left(t_{0}\right)$, which contains $x_{0}$. Put $D_{\mu}$ for that component of $\left\{(x, t): u(x, t) \geq \mu, t \leq t_{0}\right\}$ which contains $\left(x_{0}, t_{0}\right)$. Choose $\mu$ so close to 1 that $D_{\mu}$ does not contain any other component of $\Omega\left(t_{0}\right)$ but $\tilde{\Omega}\left(t_{0}\right)$. Set

$$
T_{\mu}=\overline{\partial D_{\mu}-\left\{(x, t): t=t_{0}\right\}}
$$

On $T_{\mu}$ we have $u(x, t)=\mu$ and, by Lemma $2.3($ iii),

$$
v(x, t):=u(x, t-\Delta t) \leq \mu-\delta \text { for some } \delta>0 .
$$

Hence, the set $K_{\mu-\delta / 2}:=\left\{(x, t): v(x, t)=\mu-\delta / 2, t<t_{0}\right\}$ is strictly contained in $D_{\mu}$, and the distance between $K_{\mu-\delta / 2}$ and $T_{\mu}$ on horizontal lines $\{t=$ const $\}$ is larger than some positive number $\varepsilon$. Take any unit vector $e \in \mathbf{R}^{N}$ such that

$$
x_{0}+\varepsilon e \notin \Omega\left(t_{0}\right)
$$

and define

$$
v_{\varepsilon}(x, t):=u(x-\varepsilon e, t-\Delta t)
$$

Clearly, $v_{\varepsilon}$ satisfies $(\mathrm{P})^{\prime}$ in $D_{\mu}$ and $v_{\varepsilon} \leq \mu-\delta / 2$ in $T_{\mu}$. It is, therefore, a positive lower solution of $(\mathrm{P})$ in $D_{\mu}$ and, consequently,

$$
v_{\varepsilon}(x, t) \leq u(x, t) \text { in } D_{\mu} .
$$

On the other hand, by (3.1) we have

$$
v_{\varepsilon}\left(x_{0}+\varepsilon e, t_{0}\right)=u\left(x_{0}, t_{0}-\Delta t\right)=1>u\left(x_{0}+\varepsilon e, t_{0}\right),
$$

contradicting (3.2).

This result yields the following corollary concerning the dead core $\Omega(\infty)$ of the stationary problem.

\footnotetext{
${ }^{1}$ We are indebted to Professor A. Friedman for having suggested this method to us.
} 
COROLLARY 3.2. $\Omega(\infty)$ cannot be formed in finite time.

It is clear from the initial and boundary conditions that $\Omega(t)$ is empty for small $t$. The dead core starts forming after the solution has reached the value 1 . This leads us to the following

DEFINITION. The time $\tau$ of onset of the dead core is defined as

$$
\tau=\inf \{t: \Omega(t) \text { nonempty }\}
$$

From what we know so far, the concept may be vacuous. In view of Theorem $3.1, \Omega(t)$ is empty for all $t$ if the stationary problem does not have a dead core. Criteria for the existence of a stationary dead core $\Omega(\infty)$ are found in [Ba-Sp-St]. In particular, Theorem 2.1 of that paper shows that for a dead core $\Omega(\infty)$ to exist, $g(\sigma)$ must necessarily be nondifferentiable at $\sigma=1$. Thus, for a reaction of order $p$, a dead core cannot exist if $p \geq 1$. Further results on $\Omega(\infty)$, especially its asymptotic behavior as $\lambda \rightarrow \infty$, and the regularity and convexity of $\partial \Omega(\infty)$ are discussed in [Fr-Ph], where the case of a nonmonotone $g$ is also treated.

With the help of the upper solution $\bar{u}=z(t)$ of $(2.1)$, we obtain the crude bound $\tau>\int_{0}^{1}(d u / \lambda g(u))$. If $p \geq 1$ in (A-5) this integral is infinite so that no dead core is possible for any $\lambda$. This is why we required $p<1$; we now proceed to prove the existence of a dead core and to obtain an upper bound for its time of onset. When needed for clarity we write $\Omega_{\lambda}(t)$ for $\Omega(t)$.

3.2. In [Ba-Sp-St] we showed that the stationary problem corresponding to (P), with $0 \leq p<1,{ }^{2}$ has a dead core $\Omega_{\lambda}(\infty) \subset D$ if and only if $\lambda \geq \Lambda$, where $\Lambda$ is a critical value whose dependence on the domain was studied. In short, we have

$$
\Lambda=\inf \left\{\lambda: \Omega_{\lambda}(\infty) \neq \varnothing\right\}
$$

We also showed that $\lambda>\mu$ implies $\Omega_{\lambda}(\infty) \supset \Omega_{\mu}(\infty)$ and any fixed point $x_{0}$ in $D$ necessarily belongs to $\Omega_{\lambda}(\infty)$ for $\lambda$ sufficiently large. It is convenient to introduce the definition

$$
\Lambda_{0}=\inf \left\{\lambda: x_{0} \in \Omega_{\lambda}(\infty)\right\},
$$

where the notation $\Lambda_{0}$ is intended to convey the dependence on $x_{0}$. Clearly, we have $\Lambda \leq \Lambda_{0}$.

Let us now turn to the parabolic problem (P) whose solution tends to the stationary solution as $t \rightarrow \infty$. If $\lambda<\Lambda$, then for every $x \in D$ and every finite $t$, $u(x, t)$ is strictly less than the stationary solution $u(x)$. (See Lemma 2.3(iii).) The situation changes radically if the stationary problem has a dead core. If $\lambda>\Lambda$ it seems reasonable to expect that the time-dependent problem itself has a dead core whose onset occurs at a finite time $\tau$ (see (3.3)). Moreover, if $x_{0}$ is in the interior of $\Omega(\infty)$, the dead core of the stationary problem, $x_{0}$ will also belong to the dead core $\Omega(t)$ of the time-dependent problem for $t$ sufficiently large. As we have seen previously, a point on the boundary of $\Omega(\infty)$ will not belong to the time-dependent dead core for any finite value of $t$ (Corollary 3.2). These statements will be established rigorously for many cases of problem $(\mathrm{P})$.

\footnotetext{
${ }^{2}$ If $p=0, g$ is discontinuous at $\sigma=1$ and therefore (A-1) does not hold. However, this condition is not needed in the sequel.
} 
At first we confine ourselves to the nonlinearity $g(\sigma)=(1-\sigma)_{+}^{p}$, with $0 \leq p<1$. We shall, therefore, study the parabolic problem

$$
\begin{gathered}
\partial u / \partial t-A u=\lambda(1-u)_{+}^{p} \text { in } D \times(0, \infty) \\
u(x, 0)=0 ; \quad u(\partial D, t)=0, \quad t>0 .
\end{gathered}
$$

The one and only solution of this problem is denoted by $u(x, t)$ or $u_{\lambda}(x, t)$, if needed for clarity. As $t \rightarrow \infty, u(x, t)$ tends to the unique solution $u(x)$ (or $u_{\lambda}(x)$ ) of the stationary problem

$$
-A u=\lambda(1-u)_{+}^{p}, \quad x \in D ; \quad u(\partial D)=0 .
$$

In order to state our principal result, we define for $x_{0} \in \operatorname{Int} \Omega_{\lambda}(\infty)$

$$
\tau_{0}=\inf \left\{t: x_{0} \in \Omega_{\lambda}(t)\right\} \text {. }
$$

Thus, $\tau_{0}$ is the time at which $x_{0}$ first enters the dead core of the time-dependent problem. Clearly, $\tau_{0}$ depends on $x_{0}$ and $\tau_{0} \geq \tau$.

THEOREM 3.2. Let $x_{0} \in \operatorname{Int} \Omega_{\lambda}(\infty)$ and, hence, $\lambda>\Lambda_{0}$. We then have the estimate

$$
\tau_{0} \leq \frac{1}{1-p}\left[\lambda^{1 / 1-p}-\Lambda_{0}^{1 / 1-p}\right]^{-(1-p)} .
$$

REMARK Taking the infimum over all such $x_{0}$, we obtain an inequality for the onset of the dead core:

$$
\tau \leq \frac{1}{1-p}\left[\lambda^{1 / 1-p}-\Lambda^{1 / 1-p}\right]^{-(1-p)}
$$

ProOF. We shall construct a lower solution $W(x, t)$ of $(3.4)$ such that $W\left(x_{0}, T\right)$ $=1$. It then follows that $u_{\lambda}\left(x_{0}, T\right)=1$ and, hence, $u_{\lambda}\left(x_{0}, t\right)=1$ for $t \geq T$. Hence $\tau_{0} \leq T$, and the explicit expression we shall find for $T$ will yield (3.6).

Let us define

$$
W(x, t)=Z(x)-V(t), \quad \text { where } V(t)=(1-t / T)^{1 /(1-p)}, 0<t<T,
$$

and $Z(x)$ is the solution of the stationary problem (3.5) for $\lambda=\Lambda_{0}$. Since $0 \leq$ $Z(x) \leq 1$ and $Z(\partial \Omega)=0$, we have $W(x, 0) \leq 0, W(x, t) \leq 1$ and $W(\partial \Omega, t) \leq 0$ for $0<t<T$. We also note that $W\left(x_{0}, T\right)=0$ and $W$ satisfies

$$
W_{t}-A W=\Lambda_{0}(1-Z)^{p}+V^{p} / T(1-p), \quad x \in \Omega, 0<t<T .
$$

To make $W$ a lower solution we must choose $T$ large enough so that the inequality

$$
\Lambda_{0}(1-Z)^{p}+V^{p} / T(1-p) \leq \lambda(1-Z+V)^{p}=\lambda(1-W)^{p}
$$

holds for $0 \leq 1-Z \leq 1$ and $0 \leq V \leq 1$. Taking first $1-Z>0$, we can divide by the first term on the left side of the inequality to obtain

$$
1+\alpha \varepsilon^{p} \leq \beta(1+\varepsilon)^{p}, \quad 0<\varepsilon<\infty
$$

where $\varepsilon=V /(1-Z), \alpha=\left[T(1-p) \Lambda_{0}\right]^{-1}$, and $\beta=\lambda \Lambda_{0}^{-1}>1$. We want to choose $\alpha$ (as great as possible) to satisfy (3.10). Clearly, we need $\alpha<\beta$. Denote the two sides of $(3.10)$ by $m(\varepsilon), M(\varepsilon)$, respectively. We have $m(0)<M(0)$, $\lim _{\varepsilon \rightarrow \infty} m(\varepsilon) / M(\varepsilon)=\alpha / \beta<1, M^{\prime}-m^{\prime}<0$ for $\varepsilon<\varepsilon^{*}, M^{\prime}-m^{\prime}>0$ for $\varepsilon>\varepsilon^{*}$ where

$$
\varepsilon^{*}=\alpha^{1 /(1-p)} /\left(\beta^{1 /(1-p)}-\alpha^{1 /(1-p)}\right) .
$$


Since we want $m(\varepsilon) \leq M(\varepsilon)$, it suffices to set $m\left(\varepsilon^{*}\right)=M\left(\varepsilon^{*}\right)$, which yields $\alpha^{1 /(1-p)}=\beta^{1 /(1-p)}-1$, or

$$
T(1-p)=\left[\lambda^{1 /(1-p)}-\Lambda_{0}^{1 /(1-p)}\right]^{-(1-p)} .
$$

If $T$ is so chosen then (3.9) holds for $0<1-Z \leq 1$ and $0 \leq V \leq 1$. This choice also works for $1-Z=0$, since if $T$ satisfies $(3.11)$ then $[T(1-p)]^{-1} \leq \lambda$. This completes the proof of the theorem.

In order to use estimates (3.6) and (3.7), we need some good explicit upper bounds to $\Lambda$ and $\Lambda_{0}$. Such bounds can be found in [Ba-Sp-St, Fr-Ph, and DiHe, 1]. For the sake of completeness we include here a representative result for the case $A=\Delta$.

LEMMA 3.1. Let $x_{0}$ be a given point in $D, r_{0}=\operatorname{dist}\left(x_{0}, \partial D\right), R=$ radius of largest inscribed sphere in $D$ and,

$$
c=2(1-p)^{-2}[2+(N-2)(1-p)] .
$$

Then

$$
\Lambda \leq c / R^{2}, \quad \Lambda_{0} \leq c / r_{0}^{2} .
$$

Proof. The function $v(x)=1-\left(\left|x-x_{0}\right| / r_{0}\right)^{2 /(1-p)}$ satisfies $v(\partial \Omega) \leq 0$ and $-\Delta v=c(1-v)^{p} / r_{0}^{2}$. Thus $v$ is a lower solution to (3.5) with $A=\Delta$ for $\lambda \geq c / r_{0}^{2}$. Moreover, $v\left(x_{0}\right)=1$ so that $u_{\lambda}\left(x_{0}\right)=1$ for $\lambda \geq c / r_{0}^{2}$. This proves the second inequality in (3.12) and the first follows immediately.

REMARK. The lemma also yields an asymptotic estimate for the location of the dead core when $\lambda$ is large. Indeed, for fixed $\lambda$, all points in $D$ that are further than $(c / \lambda)^{1 / 2}$ from $\partial D$ belong to the dead core. A sharp lower bound for $\operatorname{dist}(\partial D, \Omega(\infty))$ is constructed in [Ba-Sp-St] for convex domains, and more delicate asymptotic estimates can be found in [Fr-Ph].

Use of Lemma 3.1 in Theorem 3.2 gives the more explicit estimate contained in

THEOREM 3.3. Consider (3.4) with $A=\Delta$.

(a) Let $x_{0} \in D$ and let $\lambda>c / r_{0}^{2}$; then

$$
\tau_{0} \leq(1-p)^{-1}\left[\lambda^{1 /(1-p)}-\left(c / r_{0}^{2}\right)^{1 /(1-p)}\right]^{-(1-p)}
$$

(b) If $\lambda>c / R^{2}$,

$$
\tau \leq(1-p)^{-1}\left[\lambda^{1 /(1-p)}-\left(c / R^{2}\right)^{1 /(1-p)}\right]^{-(1-p)} .
$$

REMARK. Related estimates can be found in [Di-He, 2].

EXAMPLE. Let $A=\Delta, p=0$ and $D$ be the slab $0<x_{1}<1$. A simple calculation gives $\Lambda=8$ so that (3.7) yields the bound $\tau \leq 1 /(\lambda-8)$ for the time of onset of the dead core.

It is worth mentioning, perhaps only as a curiosity, that a method similar to that used in estimating blow-up times (see [Ka]) also provides an estimate for the time of onset of the dead core when $g$ is discontinuous at $u=0$. Let $A$ be the general operator of $\S 1.2$ and take

$$
g(u)= \begin{cases}1, & u<1 \\ 0, & u \geq 1\end{cases}
$$


Let $\left(\lambda_{1}, \phi_{1}\right)$ be a fundamental eigenpair of $-A \phi=\lambda \phi, \phi(\partial D)=0, \phi(x)>0$. Multiply (P) by $\phi_{1}$ and integrate over $D$ to obtain

where

$$
E^{\prime}+\lambda_{1} E=\lambda \int_{D} g(u) \phi_{1} d x
$$

$$
E(t)=\int_{D} u(x, t) \phi_{1}(x) d x .
$$

Now, for $t<\tau, u(x, t)<1$ and $g(u)=1$ so that

Hence, we find

$$
E^{\prime}+\lambda_{1} E=\lambda \int_{D} \phi_{1} d x, \quad 0<t<\tau, \quad E(0)=0 .
$$

$$
E=\frac{\lambda}{\lambda_{1}}\left[1-e^{-\lambda_{1} t}\right] \int_{D} \phi_{1} d x, \quad t<\tau .
$$

Since $E \leq \int_{D} \phi_{1} d x$, we obtain

$$
\tau \leq\left(1 / \lambda_{1}\right)\left|\log \left(1-\lambda_{1} / \lambda\right)\right|
$$

which should be compared with our previous result in the special example of a slab $\left(\lambda_{1}=\pi^{2}\right)$.

3.3. To treat more general functions $g$ satisfying (A-1)-(A-5) with $0 \leq p<1$ (in particular, $g$ may now have a jump discontinuity at $\sigma=1$ ), we propose a different method for constructing a lower solution to $(\mathrm{P})$ that reaches the value 1 for some $x$ and $t$.

Let $z(t)$ be the solution of $(2.1)$ with $\lambda=1$. For the range of $p$ under consideration,

$$
J:=\int_{0}^{1} \frac{d \sigma}{g(\sigma)}
$$

is finite and $z(t)<1$ if and only if $t<J$. Let us set

$$
\underline{u}(x, t)=z(\alpha t v(x))
$$

where $\alpha$ is a positive constant at our disposal and $v$ is a nonnegative solution of

$$
A v+q(v)=0 \quad \text { in } D, \quad v(\partial D)=0, \quad q>0 .
$$

For the time being we only require $q$ to be continuous. We denote the maximum of $v(x)$ by $v_{M}$ and the maximum of $q(v)$ for $0 \leq v \leq v_{M}$ by $q_{M}$. Note that $\underline{u}$ reaches the value 1 somewhere in $D$ when $t=J / \alpha v_{M}$. From the definition of $\underline{u}, v$ and $z$, it follows that

$$
\begin{aligned}
\underline{u}_{t}-A \underline{u} & =\alpha[v+t q(v)] z^{\prime}-\alpha^{2} t^{2} z^{\prime \prime} \sum_{i, j=1}^{N} a_{i j} v_{x_{i}} v_{x_{j}} \\
& =\alpha g[z(\alpha t v)]\left\{v+t q(v)-t^{2} \alpha g^{\prime}[z(\alpha t v)] \sum_{i, j=1}^{N} a_{i j} v_{x_{i}} v_{x_{j}}\right\} \\
& =: \alpha g[z(\alpha t v)] C(t, x ; v(x)) .
\end{aligned}
$$

For $\underline{u}$ to be a lower solution, corresponding to $\lambda$, in the time interval $\left[0, J / \alpha v_{M}\right]$, it suffices that

$$
\alpha C(t, x ; v(x)) \leq \lambda, \quad x \in \bar{D}, 0 \leq t \leq J / \alpha v_{M}
$$


which immediately gives

LEMMA 3.2. If $\alpha$ and $q$ can be chosen so that

$$
C(t, x ; v(x)) \leq C_{0}, \quad x \in \bar{D}, 0 \leq t \leq J / \alpha v_{M}
$$

then problem (P) has a dead core for $\lambda \geq \alpha C_{0}$ and the time of onset $\tau$ satisfies $\tau \leq J / \alpha v_{M}$.

It is worth noting that the monotonicity condition is not used to prove this lemma.

We shall now give more explicit examples to which Lemma 3.2 applies. For this purpose we need a result of [Pa-St].

LEMMA 3.3. Let $A=\Delta$ and let the mean curvature of $\partial D$ be positive everywhere (which is the case, for instance, for convex domains). Then

$$
|\operatorname{grad} v|^{2} \leq 2\left\{Q\left(v_{M}\right)-Q(v)\right\}
$$

where $Q(\sigma)=\int_{0}^{\sigma} q(s) d s$.

Under the conditions of this lemma we have

$$
C \leq v+t q(v)-2 t^{2} \alpha g^{\prime}[z(\alpha t v)]\left\{Q\left(v_{M}\right)-Q(v)\right\}=: \tilde{C}(t ; v(x)) .
$$

Here $\tilde{C}(t, v)$ is continuous for $0 \leq t \leq J / \alpha v_{M}$ and $v<v_{M}$, but because $g^{\prime}$ becomes infinite at $t v=J / \alpha$, it is not clear that $\tilde{C}$ remains bounded as $v \rightarrow v_{M}$. Condition (A-5), however, comes to the rescue.

LEMMA 3.4.

$$
\lim _{v \rightarrow v_{M}} g^{\prime}\left[z\left(J \frac{v}{v_{M}}\right)\right]\left\{Q\left(v_{M}\right)-Q(v)\right\}=-\frac{v_{M} p q\left(v_{M}\right)}{(1-p) J}
$$

ProOF. From (2.1) we find that

$$
\int_{z}^{1} \frac{d \sigma}{g(\sigma)}=J-t
$$

so

$$
\lim _{t \rightarrow J_{-}} z(t)=1
$$

Using (A-5) we obtain

$$
(1-p)^{-1} \lim _{t \rightarrow J_{-}} \frac{(1-z)^{1-p}}{J-t}=g_{0}(1-)=-\frac{1}{p} \lim _{t \rightarrow J_{-}} g^{\prime}(z(t))(1-z)^{1-p}
$$

and, therefore,

$$
\lim _{t \rightarrow J_{-}} g^{\prime}(z(t))[J-t]=-p /(1-p),
$$

from which the desired result follows easily. 
We conclude that $\tilde{C}$ is bounded, leading to

THEOREM 3.4. Let $A=\Delta, \partial D$ have positive mean curvature and let $\tilde{C}_{0}=$ $\sup \tilde{C}(t ; v)$ for $0 \leq t \leq J / \alpha v_{M}, 0 \leq v<v_{M}$. Then for $\lambda \geq \alpha \tilde{C}_{0}$ there exists $a$ time-dependent dead core whose onset time $\tau$ satisfies $\tau \leq J / \alpha v_{M}$.

REMARKS. 1. If $\partial D$ does not have positive mean curvature, we can inscribe a ball which, for sufficiently large $\lambda$, has a dead core with finite time of onset. By Corollary (3.1)(i) so must $D$.

2. By using a recent generalization of Lemma 3.3 by Hile and Protter [Hi-Pr], it turns out that Theorem 3.4 remains valid for the more general elliptic operator A.

APPLiCATION. Let $g(\sigma)=(1-\sigma)_{+}^{p}, 0 \leq p<1$. In this case $g^{\prime}$ is negative and $\left|g^{\prime}\right|$ is increasing, $J=1 /(1-p)$, so that

$\tilde{C}_{0} \leq v_{M}+\frac{1}{\alpha v_{M}(1-p)} q_{M}+\frac{2}{\alpha v_{M}^{2}(1-p)^{2}} \sup _{v<v_{M}}\left|g^{\prime}\left[z\left(J \frac{v}{v_{M}}\right)\right]\right|\left\{Q\left(v_{M}\right)-Q(v)\right\}$.

Since $\left|g^{\prime}\left[z\left(J v / v_{M}\right)\right]\right|=p v_{M} /\left(v_{M}-v\right)$ and $Q\left(v_{M}\right)-Q(v) \leq q_{M}\left(v_{M}-v\right)$, we obtain

$$
\tilde{C}_{0} \leq v_{M}+q_{M}(1+p) / \alpha v_{M}(1-p)^{2}
$$

and there exists a dead core for

$$
\lambda \geq \alpha v_{M}+\frac{q_{M}}{v_{M}} \frac{1+p}{(1-p)^{2}}
$$

with time of onset $\tau \leq 1 / \alpha v_{M}(1-p)$.

By making specific choices for $v$, we can use (3.15) to obtain more concrete estimates.

COROLlaRY 3.3. A sufficient condition for an isothermal pth order reaction with $A=\Delta$ to have a dead core in a domain satisfying the assumption of the theorem is

$$
\lambda>(1+p) /(1-p)^{2} v_{M},
$$

where $v$ is the solution of $\Delta v+1=0$ in $D, v(\partial D)=0$. In this case

$$
\tau \leq(1-p)^{-1}\left(\lambda-(1-p) /(1-p)^{2} v_{M}\right)^{-1} \text {. }
$$

ProOF. We have $q_{M}=1$ so, by taking $\alpha \rightarrow 0+$, we see that a dead core exists if (3.16) is satisfied. If $\lambda$ satisfies (3.16) we can choose

$$
\alpha v_{M}=\lambda-(1+p) /(1-p)^{2} v_{M}
$$

and since $\tau \leq 1 / \alpha v_{M}(1-p)$, the inequality of the corollary follows.

REMARK. An immediate consequence of (3.16) is that the critical value $\Lambda$ for the stationary problem satisfies

$$
\Lambda \leq(1+p) /(1-p)^{2} v_{M}
$$

We can also make the choice $q(v)=\lambda_{1} v$, where $\lambda_{1}$ is the fundamental eigenvalue of $-\Delta$ with Dirichlet boundary conditions and $v>0$ is a corresponding eigenfunction. Then $q_{M}=\lambda_{1} v_{M}$ and (3.15) guarantees a dead core for $\lambda>\lambda_{1}(1+p) /(1-p)^{2}$. The time of onset $\tau$ satisfies

$$
\tau \leq(1-p)^{-1}\left[\lambda-\lambda_{1}(1+p) /(1-p)^{2}\right]^{-1} .
$$


REMARKS 1. Lower bounds for $v_{M}$ are found, for example, in [Ba].

2. The bounds of Corollary 3.3 and the earlier bounds of Theorem 3.3 are not comparable, in the sense that, depending on the domain, either can provide a better estimate than the other.

\section{Rearrangements and isoperimetric inequalities.}

4.1. In this section we use rearrangement techniques to derive estimates which depend only on the measure of $D$.

First, let us introduce the following notation:

$M$ is the measure of $D$.

$D(\mu, t)=\{x \in D: u(x, t) \geq \mu\}$.

$m(\mu, t)=\operatorname{meas}\{D(\mu, t)\}$.

$\tilde{u}(a, t)=\sup \{\mu: m(\mu, t) \geq a\}$ is the decreasing rearrangement of $u$.

Moreover, we write

$$
H(a, t)=\int_{D(\tilde{u}(a, t), t)} u(x, t) d x .
$$

In terms of our earlier notation, we have $D(0, t)=D, D(1, t)=\Omega(t)$.

DEfinition. Problem (P)* stands for problem (P) with $A=\Delta$ in the ball $D^{*}$ of the same measure as $D$. We shall indicate the quantities corresponding to $(\mathrm{P})^{*}$ by $*$.

The following properties of rearrangements are easily derived (see [Ba, Ta], for instance).

(A) For any continuous function $\psi>-\infty$,

$$
\int_{0}^{a} \psi[\tilde{u}(\alpha, t)] d \alpha=\int_{D(\tilde{u}(a, t), t)} \psi[u(x, t)] d x .
$$

(B) If $u(\cdot, t) \in H_{0}^{1}(D)$ and $u_{t}(\cdot, t) \in L^{2}(D)$ solve $A u+\lambda g(u)-u_{t}=0$ in $D$, then

$$
q(a) \tilde{u}_{a}(a, t)+\int_{D(\tilde{u}(a, t), t)}\left[\lambda g(u)-u_{t}\right] d x \geq 0 \quad \text { in }(0, M),
$$

where $q^{1 / 2}(a)$ is the surface area of the sphere of volume $a$. Equality holds for the solution $u^{*}$ in $D^{*}$ with $A=\Delta$.

(C) For any domain $B \subset D$ of volume $a$, we have

$$
\int_{B} u(\cdot, t) d x \leq \int_{D(\tilde{u}(a, t), t)} u(\cdot, t) d x=\int_{0}^{a} \tilde{u}(\alpha, \cdot) d \alpha .
$$

A further property of the rearrangement, already derived in [Ba] under stronger regularity conditions, is expressed in

LEMMA 4.1. Let $u: D \times \mathbf{R}^{+} \rightarrow \mathbf{R}$ be a measurable function. Suppose $u(x, \cdot)$ is monotone and $u_{t}(\cdot, t) \in L^{1}(D)$. Then

$$
\int_{D(\tilde{u}(a, t), t)} u_{t}(x, t) d x=H_{t}(a, t) \text { for almost every } t .
$$

ProOF. Let $\left\{t_{n}\right\}_{n=1}^{\infty}$ be any sequence such that $t_{n} \rightarrow t$ as $n \rightarrow \infty$. Put, for short, $D_{0}:=D(\tilde{u}(a, t), t)$ and $D_{n}=D\left(\tilde{u}\left(a, t_{n}\right), t_{n}\right)$. By Lebesgue's dominated convergence theorem

$$
\int_{D_{0}} u_{t} d x=\lim _{n \rightarrow \infty} \frac{\int_{D_{0}} u\left(x, t_{n}\right) d x-\int_{D_{0}} u(x, t) d x}{t_{n}-t}
$$


Since meas $D_{0}=$ meas $D_{n}=a$, it follows from (C) that

$$
\int_{D_{0}} u\left(x, t_{n}\right) d x \leq \int_{D_{n}} u\left(x, t_{n}\right) d x=\int_{0}^{a} \tilde{u}(\alpha, t) d \alpha .
$$

For $t_{n} \downarrow t$ we conclude from (4.3) and (4.2) that

$$
\int_{D_{0}} u_{t} d x \leq \varlimsup_{n \rightarrow \infty} \frac{H\left(a, t_{n}\right)-H(a, t)}{t_{n}-t} .
$$

Similarly, for $t_{n} \uparrow t$,

$$
\int_{D_{0}} u_{t} d x \geq \varliminf_{n \rightarrow \infty} \frac{H\left(a, t_{n}\right)-H(a, t)}{t_{n}-t} .
$$

Since $u(x, \cdot)$ and, therefore, $H(a, \cdot)$ are monotonic, $H_{t}(a, t)$ exists for almost all $t$ and the assertion is now obvious.

This lemma applied to (B) yields

LEMMA 4.2. Let $u(x, t)$ be a solution of $(\mathrm{P})$ and $H(a, t)$ be defined as above. Then $H$ satisfies the differential inequality

$$
q(a) H_{a a}(a, t)+\lambda \int_{0}^{a} g(\tilde{u}) d \alpha-H_{t}(a, t) \geq 0 \quad \text { in }(0, M)
$$

and the boundary and initial conditions $H(0, t)=0, H_{a}(M, t)=0$ and $H(a, 0)=0$. Equality holds for the solution $u^{*}$ of problem $(\mathrm{P})^{*}$.

REMARK. If $\partial D \in C^{2}$ then the same techniques together with the strong maximum principle indicate that $H_{a a}(M, t)$ exists and that (4.5) remains valid for $a=M$ and almost all $t$.

We shall also make use of a slightly more general version of a maximum principle for the one-dimensional heat equation [Pr-We, p. 160]. For the sake of completeness we shall give a proof following exactly the one of Protter and Weinberger.

LEMMA 4.3. Let $B \subset(0, M) \times \mathbf{R}^{+}$be a bounded domain, let $v(a, t)$ be a continuous function on $B$ such that $v_{a}$ is continuous, $v_{a a}$ exists for almost all $a$ and $v_{t}$ exists for almost all $t$. Suppose

$$
q(a) v_{a a}(a, t)-v_{t}(a, t) \geq 0 \text { a.e. in } B .
$$

Then $v$ cannot have a maximum at an interior point unless $v \equiv$ const. in $B$.

PROOF. Let $M=\sup _{\partial B} v$ and suppose $v$ takes its maximum $M_{1}>M$ at an interior point $\left(a_{0}, t_{0}\right) \in B$. Assume that $B$ is contained in the strip $\left\{a_{1} \leq a \leq\right.$ $\left.a_{1}+l\right\}$. We now consider the function

$$
\tilde{v}(a, t)=v(a, t)+\frac{M_{1}-M}{2 l^{2}}\left(a-a_{0}\right)^{2}
$$

which has the following properties:

(i) $\tilde{v}(a, t)<M+\left(M_{1}-M\right) / 2<M_{1}$ on $\partial B$.

(ii) $\tilde{v}\left(a_{0}, t_{0}\right)=M_{1}$.

(iii) $q(a) \tilde{v}_{a a}-\tilde{v}_{t}=q(a)\left[v_{a a}+\left(M_{1}-M\right) / l^{2}\right]-v_{t}>0$ in $B$.

In view of (i) and (ii), $\tilde{v}$ takes its maximum at an interior point. If $\tilde{v}_{a a}$ and $\tilde{v}_{t}$ exist at this point, we have there $\tilde{v}_{a a} \leq 0$ and $\tilde{v}_{t}=0$, obviously a contradiction to (iii). 
Otherwise, consider the set $\Gamma_{\nu}=\{(a, t): \tilde{v}(a, t)=\nu\}$. If $\nu$ is near the maximum $\tilde{v}_{m}$ of $\tilde{v}$ in $B, \Gamma_{\nu}$ is a closed curve. Denote by $\left(a_{\nu}, t_{\nu}\right)$ a point on $\Gamma_{\nu}$ with the smallest $t$-coordinate. By assumption, there is a $\nu_{0}$ in $\left(\tilde{v}_{m}-\delta, \tilde{v}_{m}\right)$ such that $\tilde{v}_{a a}$ and $\tilde{v}_{t}$ exist at $P_{0}:=\left(a_{\nu_{0}}, t_{\nu_{0}}\right)$. At such a point we have $\tilde{v}_{t}\left(P_{0}\right) \geq 0$ and $\tilde{v}_{a a}\left(P_{0}\right) \leq 0$, contradicting (iii).

4.2. We now proceed to our main theorems of this section.

THEOREM 4.1. Let $u$ and $u^{*}$ be the solutions of problems $(\mathrm{P})$ and $(\mathrm{P})^{*}$, respectively, and suppose $\partial D \in C^{2}$. Then for all positive $t$,

$$
\max _{x \in D} u(x, t) \leq \max _{x \in D^{*}} u^{*}(x, t) .
$$

Moreover,

$$
\operatorname{meas} \Omega(t) \leq \operatorname{meas} \Omega^{*}(t) .
$$

PROOF. Let us introduce the notation

$$
E(a, t)=\lambda \int_{D(\tilde{u}(a, t), t)} g(u) d x=\lambda \int_{0}^{a} g(\tilde{u}(\alpha, t)) d \alpha,
$$

$\delta H(a, t):=H(a, t)-H^{*}(a, t)$ and $\delta E(a, t):=E(a, t)-E^{*}(a, t)$. Suppose that (4.7) is not true for all $t$. Then there is a $t_{0}>0$ such that $\max _{x \in D} u\left(x, t_{0}\right)>$ $\max _{x \in D^{*}} u^{*}\left(x, t_{0}\right)$ or, equivalently, $\tilde{u}\left(0, t_{0}\right)>\tilde{u}^{*}\left(0, t_{0}\right)$. Since both $\tilde{u}$ and $\tilde{u}^{*}$ are continuous, there exists a neighborhood $N$ of $\left(0, t_{0}\right)$ such that

$$
\begin{cases}\tilde{u}(a, t)>\tilde{u}^{*}(a, t) & \text { in } N \\ \tilde{u}(a, t)=\tilde{u}^{*}(a, t) & \text { in } \partial N^{-}:=\partial N \cap\left\{(a, t): a>0, t<t_{0}\right\} .\end{cases}
$$

Consequently,

$$
\delta H>0 \text { in } N \text {. }
$$

Let us first assume that $g^{\prime}(\sigma)<0$ in $(0,1)$. Then $\delta E<0$ in $N$ and, in particular, $\delta E<0$ on $\partial N^{-}$, and, by the continuity of $\delta E$, we have $\delta E<0$ in a larger region $N^{\prime}$ containing $N \cup \partial N^{-}$. By Lemma 4.2

$$
q(a) \delta H_{a a}-\delta H_{t} \geq 0 \text { in } N^{\prime} \text {. }
$$

It is clear from (4.10) and from $\delta H(a, 0)=\delta H(0, t)=0$ that the maximum of $\delta H$ in $N^{\prime}$ is attained on $\partial N^{-}$, say at $P$. Because of (4.11) and Lemma 4.3,P cannot be in the interior of $N^{\prime}$, hence it has to be in $\{a=M\}$. According to the remark after Lemma 4.2 and our regularity assumption on $\partial D$, we must have

$$
q(M) \delta H_{a a}(P)-\delta H_{t}(P)>0,
$$

which contradicts the fact that $\delta H_{a a}(P) \leq 0$ and $\delta H_{t}(P)=0 .^{3}$ Thus, $\tilde{u}(0, t) \leq$ $\tilde{u}^{*}(0, t) \forall t \geq 0$. In the general case, we approximate $g(\sigma)$ by functions $g^{\varepsilon}(\sigma)$ such that $d g^{\varepsilon} / d \sigma<0$ in $(0,1), g^{\varepsilon}(\sigma) \geq g(\sigma)$ and $g^{\varepsilon}(\sigma)-g(\sigma) \leq \varepsilon$, establish the result for $\tilde{u}^{\varepsilon}$ and $\tilde{u}^{\varepsilon^{*}}$ and let $\varepsilon \rightarrow 0$, using Lemma 2.7. The second assertion follows from exactly the same argument. Suppose meas $\Omega\left(t_{0}\right)>$ meas $\Omega^{*}\left(t_{0}\right)$ for $t_{0}>0$. Then

$$
\tilde{u}\left(a, t_{0}\right)=\tilde{u}^{*}\left(a, t_{0}\right) \quad \text { in }\left(0, \text { meas } \Omega^{*}\left(t_{0}\right)\right]
$$

\footnotetext{
${ }^{3} \mathrm{~A}$ slight modification as in Lemma 4.3 is needed if $\delta H_{t}(P)$ is not defined.
} 
and

$$
\tilde{u}\left(a, t_{0}\right)>\tilde{u}^{*}\left(a, t_{0}\right) \quad \text { in }\left(\operatorname{meas} \Omega^{*}\left(t_{0}\right), \bar{a}\left(t_{0}\right)\right), \bar{a}\left(t_{0}\right) \leq M .
$$

There exists, therefore, a neighborhood $\tilde{N}$ of $\left(\operatorname{meas} \Omega^{*}\left(t_{0}\right), t_{0}\right)$ such that $\delta u>0$ in $\tilde{N}$. As before, this assumption leads to a contradiction. The theorem is now established.

REMARK. The corresponding result for the stationary problem has already been derived in [Ba-Sp-St].

As an immediate consequence we have

COROllary 4.1. For $D$ to have a dead core at time $t$ it is necessary that $(\mathrm{P})^{*}$ have a dead core at time $t$ for $D^{*}$.

EXAMPLE. For an isothermal $p$ th-order reaction with $A=\Delta$, a straightforward computation carried out in [Ba-Sp-St] shows that $\Omega^{*}(\infty)$ exists only for $p \in[0,1)$ and for $D^{*}$ sufficiently large. More precisely we have, writing $R$ for the radius of $D^{*}$ and $\omega_{N}$ for the volume of the unit sphere in $\mathbf{R}^{N}$,

$$
\text { meas } \Omega^{*}(\infty)=\omega_{N}\left\{R-\frac{2}{\lambda(1-p)} \sqrt{2 N(1-p)+4 p}\right\}^{N}
$$

and by (4.8) and Theorem 3.1,

$$
\text { meas } \Omega(t) \leq \operatorname{meas} \Omega^{*}(t) \leq \operatorname{meas} \Omega^{*}(\infty) .
$$

ACKNOWLEDGEMENT. This research was performed while one of the authors (C.B.) was visiting the University of Delaware. She would like to express her thanks to the Department of Mathematical Sciences and to the Visiting Women's Scholar Fund for their support. The research of the second author (I.S.) was supported by a National Science Foundation grant.

\section{REFERENCES}

[Ar] R. Aris, The mathematical theory of diffusion and reaction in permeable catalysts, Clarendon Press, Oxford, 1975.

[Ba] C. Bandle, Isoperimetric inequalities and applications, Pitman, New York, 1980.

[Ba-Sp-St] C. Bandle, R. P. Sperb and I. Stakgold, Diffusion-reaction with monotone kinetics, Nonlinear Anal. 8 (1984), 321-333.

[De-He] J. Deuel and P. Hess, Nonlinear parabolic boundary value problems with upper and lower solutions, Israel J. Math. 29 (1978), 92-104.

[Di-He, 1] J. Diaz and J. Hernandez, On the existence of a free boundary for a class of reaction-diffusion systems, SIAM J. Math. Anal. (to appear).

[Di-He, 2] _ _ Some results on the existence of free boundaries for parabolic reaction-diffusion systems, Proc. Fifth Internat. Conference on Trends in Theory and Practice of Nonlinear Differential Equations, Dekker, New York, 1984.

[Fr-Ph] A. Friedman and D. Phillips, The free boundary of a semilinear elliptic equation, Trans. Amer. Math. Soc. 282 (1984), 153-182.

[Hi-Pr] G. N. Hile and M. H. Protter, Gradient bounds for weakty coupled nonlinear elliptic systems, Applicable Anal. 16 (1983), 51-70.

[Ka] S. Kaplan, On the growth of solutions of quasi-linear parabolic equations, Comm. Pure Appl. Math. 16 (1963), 305-330.

[Pa-St] L. E. Payne and I. Stakgold, Nonlinear problems in nuclear reactor analysis, Nonlinear Problems in the Physical Sciences and Biology, Lecture Notes in Math., vol. 322, Springer-Verlag, Berlin and New York, 1973, pp. 298-307. 
[Pr-We] M. H. Protter and H. Weinberger, Maximaum principles in differential equations, PrenticeHall, Englewood Cliffs, N.J., 1967.

[St] I. Stakgold, Estimates for some free boundary vakue problems, (Proc. Conf. Ordinary and Partial Differential Equations), Lecture Notes in Math., vol. 846, Springer-Verlag, Berlin and New York, 1981, pp. 333-346.

[Ta] G. Talenti, Elliptic equations and rearrangements, Ann. Scuola Norm. Sup. Pisa Cl. Sci. 3 (1976), 697-718.

[Tr] F. Treves, Basic linear partial differential equations, Academic Press, New York, 1975.

DePartment OF MATHEMATICS, UNIVERSity OF BASEL, BASEl, SWITZERLAND

Department of Mathematical Sciences, University of Delaware, Newark, DELAWARE 19716 